\title{
Does Low IgA in Human Milk Predispose the Infant to Development of Cow's Milk Allergy?
}

\author{
KIRSI-MARJUT JÄRVINEN, SEPPO T. LAINE, ANNA-LIISA JÄRVENPÄÄ, AND \\ HANNA K. SUOMALAINEN
}

\begin{abstract}
Helsinki University Central Hospital, Department of Dermatology, FIN-00029 HUCH, Helsinki [K.-M.J., H.K.S.]; Tampere University Hospital, Department of Clinical Microbiology, Division of Clinical Immunology, FIN-33521 Tampere [S.T.L.]; and Helsinki City Maternity Hospital, FIN-00610 Helsinki,
\end{abstract} Finland [A.-L.J.]

\begin{abstract}
We sought a relationship between total and cow's milkspecific IgA levels in colostrum and human milk and subsequent development of cow's milk allergy (CMA) in the breast-fed infant. The study included 87 nursing mothers and their infants (age, $2 \mathrm{~d}$ to $7 \mathrm{mo}$ ), followed prospectively up to $1 \mathrm{y}$. At $1 \mathrm{y}, 48$ mothers $(69 \%$ with an atopic constitution) had an infant with CMA, verified by clinical cow's milk challenge, eight (38\% with an atopic constitution) had a baby who had had protracted infantile colic but no CMA (disease control group), and 31 (23\% with an atopic constitution) had a healthy infant. Total breastmilk IgA was measured by radial immunodiffusion, and IgA antibodies to cow's milk were measured by ELISA during the breast-feeding period. The levels of total and cow's milk-specific IgA antibodies in colostrum and human milk were significantly lower in the mothers whose baby later developed CMA [estimated third day value, $0.38 \mathrm{~g} / \mathrm{L}$ (95\% confidence interval, $0.24-$ $0.82)]$ than in the ones whose infant remained healthy or had had infantile colic but not CMA $[0.82 \mathrm{~g} / \mathrm{L}$ (95\% confidence interval, $0.99-1.51) ; p<0.05]$. The infants developed CMA significantly
\end{abstract}

\section{ABSTRACT}

more often if the concentration of total $\mathrm{IgA}$ antibodies in milk was $<0.25 \mathrm{~g} / \mathrm{L}$, when measured between $6 \mathrm{~d}$ and 4 wk postpartum [sensitivity, 0.55; specificity, 0.92; odds ratio, $14.7(95 \%$ confidence interval, 3.1-70.2); $p<0.001]$. The levels of cow's milk-specific IgA positively correlated with the levels of total IgA but not with the development of CMA in the infant. The levels of total or cow's milk-specific IgA did not correlate with maternal atopy. IgA antibodies in colostrum and human milk may prevent antigen entry at the intestinal surface of the breastfed infant. A low IgA content in human milk may lead to defective exclusion of food antigens and thus predispose an offspring to develop food allergies. (Pediatr Res 48: 457-462, 2000)

CMA, cow's milk allergy

\section{Abbreviations}

$\mathbf{E U}$, enzyme immunoassay unit

$\operatorname{sIgA}$, secretory IgA

CI, confidence interval
The effect of breast-feeding in reducing the incidence of atopic disease and CMA in the first years of life still remains to be settled (1-8). Some studies have even reported an increased risk of development of atopic eczema and food allergies in breast-fed infants (9-12). Apart from a couple of studies, there has been no support for the view that some mothers produce milk deficient in certain immunologic factors, and so predispose their infants to food allergy (13-15).

Much of the passive protection conferred by antimicrobial substances in human milk is caused by the IgA present almost entirely in dimeric or secretory form (16). It is present in highest concentrations in the first few days postpartum (in the

Received August 19, 1999; accepted April 19, 2000.

Correspondence and reprint requests: Hanna Suomalainen, M.D., Ph.D., Helsinki University Central Hospital, Skin and Allergy Hospital, Department of Dermatology, P.O. Box 160, FIN-00029 HUCH, Helsinki, Finland.

Supported (personal grant) by the Finnish Medical Foundation, the Yrj246š Jahnsson Foundation and the Ida Montin Foundation (K.-M.J.). colostrum), and then falls away progressively to a basal level of 0.2 to $0.3 \mathrm{~g} / \mathrm{L}(13,14)$. sIgA is more resistant to acid conditions and to the proteolytic activity of gut enzymes than serum IgA (17), and is found intact in significant quantities in the feces $(17,18)$. The sites of action of the ingested IgA are restricted because this Ig apparently cannot be absorbed from the infant gut in significant quantities (19). Milk Igs have been shown to be absorbed for a short period of 18 to $24 \mathrm{~h}$ after birth (18), although the importance of this to the infant is unknown.

A number of sIgA antibodies to common food antigens, bacteria, and viruses to which the mother has been exposed have been described in human milk $(13,14,20-22)$. These antibodies appear to be particularly important during the first few days of life, when the infant's mucosal IgA production is deficient $(21,23)$. After ingestion, maternal milk IgA antibodies have been suggested to passively protect the infant by binding to IgA receptors on the luminal surface of enterocytes and thereby decreasing or preventing antigen entry across the 
immature gastrointestinal epithelium (24). In an experimental model, Walker et al. (25) have shown that intestinal antibodies can prevent resorption of native proteins and instead increase the uptake of degraded material, probably by binding the antigens and exposing them to the intestinal enzymes. IgA antibodies might also play a role in excluding potential food allergens from human milk by forming immune complexes that could be phagocytosed by human milk macrophages (24). In previous preliminary studies, samples of colostrum from mothers whose infants later showed symptoms of CMA (13) or developed CMA verified by oral challenge (14) had low total IgA contents $(13,14)$ and low levels of specific IgA to cow's milk (13).

The purpose of this study was to determine the content of total and cow's milk-specific IgA in colostrum and human milk in relation to the subsequent development of CMA in the breast-fed infant.

\section{METHODS}

Subjects. The subjects were enrolled in the study between April 1995 and May 1998. The study population comprised 87 breast-feeding mothers and their infants. Of these, 27 infants were followed prospectively from birth because of their high genetic risk of food allergy (at least one sibling with severe food allergy diagnosed by elimination and open challenge). A further 17 infants were defined as being at moderate risk for developing food allergy because they had at least one parent with atopic constitution. The remaining four infants were considered as having a low risk for food allergy because they had no allergic or atopic first-degree relatives. An additional 25 infants were included in the study as young infants because of symptoms suggestive of CMA. All the infants were aged from $2 \mathrm{~d}$ to $7 \mathrm{mo}$. The infants that were followed up prospectively were included in the study immediately after birth, and the first milk sample was received from their mothers at the median age of $4 \mathrm{~d}$ (range, 2 to $44 \mathrm{~d}$ ). The ones recruited because of symptoms suggestive of food allergy were aged 4.7 mo (median; range, 1.8 to $6.8 \mathrm{mo}$ ) at the time of inclusion into the study. All the infants were born at full-term, and had neither chronic diseases apart from allergy nor infection while visiting the clinic. They were followed up during the first year of life to detect any symptoms suggestive of food allergy.

The infants and the mothers of the infants having symptoms suggestive of food allergy were put on an elimination diet: foods suspected to cause the symptoms in the infant, e.g. cow's milk, cereals (rye, wheat, oats, and barley), egg, and fish, were eliminated from the mother's and the infant's diets. The healthy infants had diets appropriate for their ages. All the atopic mothers were symptom-free (no hayfever, eczema, urticaria, or asthma) during the breast-feeding period. The mothers had no medication during the study, and those who had had mastitis during the preceding $4 \mathrm{wk}$ were excluded.

The study protocol was approved by the ethics committees of the Skin and Allergy Hospital of the Helsinki University Central Hospital and the City of Helsinki. Informed consent was obtained from the mothers for collection of breast-milk samples and for longitudinal follow-up of their children.
Skin-prick tests. Skin-prick tests were performed with a commercial cow's milk extract (Soluprick, Allergologisk Laboratorium A/S, Horsholm, Denmark) on the volar side of the forearm, using a 1-mm, one-peak lancet with shoulder. Histamine dihydrochloride (10 mg/mL; Soluprick) was used as a positive control. Reactions were red at $15 \mathrm{~min}$ and regarded as positive if the mean diameter of the wheal was $\geq 3 \mathrm{~mm}$, the negative control was $0 \mathrm{~mm}$ and the positive control $\geq 5 \mathrm{~mm}$ at the same time.

Cow's milk and cereal challenge protocol. The diagnosis of CMA and cereal allergy was confirmed by open food challenge, because placebo reactions have been shown to be rare in infants (26). After elimination of cow's milk or cereals for 2 to $4 \mathrm{wk}$ and amelioration of symptoms, patients were admitted, in the absence of any other disease or infection, for an oral cow's milk or cereal challenge. The challenge was started with a drop of adapted formula (Tutteli, Valio Ltd., Helsinki, Finland), containing $1.4 \mathrm{~g} / \mathrm{L}$ of beta-lactoglobulin (27) or cereal (rye, wheat, oats, or barley) on the skin or lips. On day 1, food was given in increasing doses $(1,10,50$, and $100 \mathrm{~mL}$ of adapted formula, or 1,5 , and $10 \mathrm{~g}$ of cereal) at 1-h intervals. On day 2, $200 \mathrm{~mL}$ of milk or $20 \mathrm{~g}$ of cereal was given, followed by milk or cereal intake appropriate for the infant's age. The challenge was continued for $1 \mathrm{wk}$ to see any late-phase reactions. If any adverse reaction appeared, the challenge was stopped, and the patients were examined by an experienced pediatrician or a dermatologist. The diagnosis was based on typical symptoms, their amelioration on cow's milk elimination diet, and reappearance on cow's milk-containing diet. Symptoms regarded as positive were urticaria, flair of eczema, vomiting, and diarrhea. Additional symptoms recorded were abdominal pain and restlessness in addition to the symptoms of the upper airways and respiratory tract such as watery rhinorrhea and wheezing.

Collection of human milk specimens. The first human milk sample was taken from the mothers during the first 2 wk after delivery, and then at the ages of 1, 3, and 6 mo of the infant. Additional samples were collected at the time of onset of the symptoms of CMA. In seven mothers, milk samples were collected frequently every third day to determine the precise course of the IgA levels in human milk. An average of 2.6 samples (range, 1 to 12 ) was obtained per mother during the course of breast-feeding.

The breasts were washed with warm water before collecting the milk with a manual breast pump. Immediately after collection, human milk samples, 20 to $50 \mathrm{~mL}$ in volume, were centrifuged at $1400 \times g$ at room temperature for $15 \mathrm{~min}$ to separate the fat layer. Subsequently, the fat was removed, and the supernatant was collected, frozen, and stored at $-70^{\circ} \mathrm{C}$ until further determination of human milk IgA.

Detection of human milk total $\operatorname{Ig} A$ and $\operatorname{Ig} A$ antibodies to cow's milk. After thawing, human milk samples were diluted $1: 5$ in PBS solution ( $\mathrm{pH} 7.4$ ) including $0.05 \%$ Tween 20 . The rest of the fatty layer was removed by centrifugation at $17,000 \times g$ for $10 \mathrm{~min}$ at $4^{\circ} \mathrm{C}$. Supernatants were used for determination of IgA antibodies to cow's milk, and they were further diluted 1:2 for the measurement of total IgA. Breast milk total IgA was determined by radial immunodiffusion, 
using LC-Partigen IgA immunodiffusion plates with N Protein Standard SL as a calibrator according to the manufacturer's instructions (Dade Behring, Marburg, Germany). Results are expressed as grams per liter.

Specific IgA antibodies to cow's milk were determined by a modification of the methods described by Knoflach et al. (28). Dried whole cow's milk powder (Valio Ltd, Helsinki, Finland) was dissolved in sodium carbonate buffer $\left(0.1 \mathrm{M} \mathrm{Na}_{2} \mathrm{CO}_{3}, 1\right.$ $\mathrm{mM} \mathrm{MgCl} 2, \mathrm{pH} 9.8$ ) to give a stock solution of $1 \mathrm{mg}$ protein/ $\mathrm{mL}$. Microtiter plates (Nunc-Immuno Plate MaxiSorp, Nunc A/S, Roskilde, Denmark) were coated with $100 \mu \mathrm{L}$ of antigen solution diluted to a protein concentration of $0.3 \mu \mathrm{g} / \mathrm{mL}$. The plates were incubated at $37^{\circ} \mathrm{C}$ for $3 \mathrm{~h}$. The wells were washed three times with PBS-Tween 20. One hundred microliters of test milk supernatant and a dilution series of a positive patient's serum forming a standard curve were added to the wells and incubated at room temperature for $16 \mathrm{~h}$. After the wells had been washed three times with PBS-Tween 20, $100 \mu \mathrm{L}$ of alkaline phosphatase-conjugated anti-human IgA antiserum (Dako A/S, Glostrup, Denmark) diluted 1:1000 in PBS-Tween 20 was added to the wells and incubated at $37^{\circ} \mathrm{C}$ for $2 \mathrm{~h}$. After incubation, the wells were washed with PBS-Tween 20, and $100 \mu \mathrm{L}$ of fresh substrate solution (para-nitrophenyl phosphate $1 \mathrm{mg} / \mathrm{mL}$ in diethanolamine- $\mathrm{MgCl}_{2}$ buffer, $\mathrm{pH} 9.8$ ) was added to each well and incubated at $37^{\circ} \mathrm{C}$ for $30 \mathrm{~min}$. At the end of incubation, $100 \mu \mathrm{L}$ of $1 \mathrm{M} \mathrm{NaOH}$ was added to the wells to stop the reaction. Absorbances were determined at a wavelength of $405 \mathrm{~nm}$, and the specific IgA concentrations as EU per milliliter were read from a standard curve.

Statistical analysis. The results were analyzed by an experienced statistician. The data were analyzed using ANOVA for repeated measurements with the mothers as the clusters. Because the content of IgA was skewed, logarithmic transformation was applied to the values of IgA before model fitting. Because the content of IgA drops faster in the beginning and then almost levels out, the logarithm of the age of the baby was used as such as an explanatory variable. In the analysis, the starting model was as follows: $\ln ($ age $)$ as the random and systemic part; $\ln (\operatorname{Ig} \mathrm{A}) \sim \ln ($ age $) *(1$ allergy +1 mother's atopy +1 symptoms), where 1 character is an indicator variable for character. The unimportant terms (significance $>5 \%$ ) were excluded. The models were compared by ANOVA. The Kruskal-Wallis test was used to determine the statistical significance of differences between ages.

The $\chi^{2}$ test was applied to determine differences in proportions. The analyses were performed using S plus 4 software (MathSoft, Inc., Seattle, WA, U.S.A..) and StatView 4.0 software (Abacus Concepts, Inc., Berkeley, CA, U.S.A.).

Table 1. Fixed effects estimates

\begin{tabular}{lrcr}
\hline & Value & Approx. SE & $z$ ratio $(\mathrm{C})$ \\
\hline Intercept & 0.199 & 0.109 & 1.838 \\
Dg & -1.028 & 0.321 & -3.207 \\
$\ln$ (Age) & -0.361 & 0.027 & -13.158 \\
Dg: $\ln$ (Age) & 0.234 & 0.071 & 3.323 \\
\hline
\end{tabular}

The estimation of the third day value for total IgA in milk is based on this model, when describing the logarithm of $\operatorname{IgA}$.

\section{RESULTS}

Clinical characteristics of the study population. During the follow-up, 48 mothers (69\% with an atopic constitution) had an infant that had developed CMA verified by clinical cow's milk challenge (14 infants with a history of protracted colic), eight (38\% with an atopic constitution) had a baby who had had protracted infantile colic but who had a negative cow's milk challenge (disease control group), and 31 (23\% with an atopic constitution) had a healthy infant. Challenge-proven hypersensitivity against rye, wheat, oats, and barley was detected in 32 of $48(67 \%)$ infants with CMA. The duration of exclusive breast-feeding was comparable in the infants with CMA, median was $3.5 \mathrm{mo}$ (range, $0-7 \mathrm{mo}$ ); in the infants in the disease control group, the median was 3.0 mo (range, 1 wk to $6 \mathrm{mo}$ ); and in the healthy infants, the median was $3.0 \mathrm{mo}$ (range, $1 \mathrm{wk}$ to 6 mo). Of the 48 infants with CMA, 35 (73\%) had been exclusively breast-fed at the time of appearance of symptoms of CMA. The median age at the time of the appearance of the symptoms of CMA was $1.3 \mathrm{mo}$ (range, $4 \mathrm{~d}$ to $4 \mathrm{mo}$ ) and at the time of the clinical challenge 4.9 mo (range, 4.0-5.9 mo). An atopic constitution of the mother correlated with the development of CMA in the infant ( $p=0.0003 ; \chi^{2}$ test).

The reaction to a skin-prick test with cow's milk was positive in 15 of $48(31 \%)$ patients with CMA. In clinical cow's milk challenge, 26 (54\%) infants manifested CMA with immediate and $22(46 \%)$ with delayed reactions. Twelve children showed local urticaria immediately after introduction of a drop of cow's milk on their skin or lips. Of the rest, 20 children had eczema, five had gastrointestinal symptoms, and 11 reacted both cutaneously and gastrointestinally to peroral administration of cow's milk. Four children showed upper airway symptoms (acute middle ear infection, allergic rhinitis, or wheezing) in addition to other symptoms of CMA.

Total and cow's milk-specific IgA levels in human milk. Colostrum samples (days 2-5) were received from seven mothers with an infant who subsequently developed CMA and from 22 mothers of healthy infants. ANOVA for repeated measurements gave an estimated (geometric mean) value for $\operatorname{IgA}$ on the third day of $0.38 \mathrm{~g} / \mathrm{L}(95 \% \mathrm{CI}, 0.24-0.82 \mathrm{~g} / \mathrm{L})$ for those mothers whose baby developed CMA and $0.82 \mathrm{~g} / \mathrm{L}(95 \% \mathrm{CI}$, $0.99-1.51 \mathrm{~g} / \mathrm{L})$ for those mothers whose infant remained healthy or had infantile colic but not CMA ( $p<0.05$; Fig. 1, Table 1). The levels of IgA antibodies dropped in both groups during the course of breast-feeding, but owing to the lower starting value, the drop was smaller and statistically insignificant in the mothers whose babies developed allergy (Fig. 1). The infants developed CMA significantly more often if the concentration of total $\operatorname{IgA}$ antibodies in milk was $<0.25 \mathrm{~g} / \mathrm{L}$, when measured between $6 \mathrm{~d}$ and 4 wk postpartum [sensitivity, 0.55; specificity, 0.92; odds ratio, 14.7 (95\% CI, 3.1-70.2); $p<$ $0.001]$. This time range was selected for a number of reasons. First, the levels of IgA have become more steady after the colostral phase, and second, the ages of the healthy and CMA infants were comparable during this period. Third, this lactational phase was considered the best for the assessment of infants at high risk of food allergy. There was no statistically 


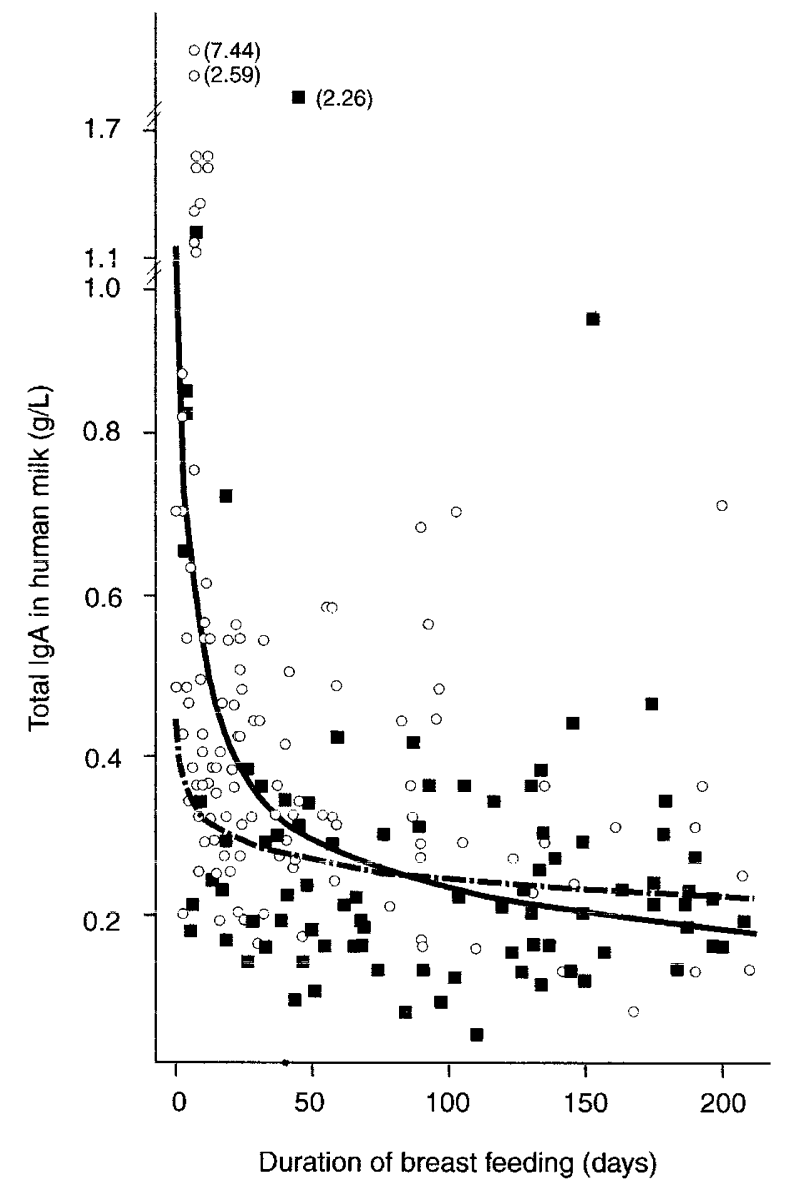

Figure 1. Concentration of total IgA in the milk of mothers with a healthy infant (open circles) and in those with a cow's milk-allergic infant (closed squares) as a function of lactational duration. Solid and broken lines represent the modeled geometric means in mothers with a healthy infant and in those with an infant with CMA, respectively. Separate models were used for both groups, and they differ significantly from each other.

significant difference in the levels of total IgA antibodies in the mothers of infants with immediate-type or delayed-type reaction.

The levels of cow's milk-specific IgA antibodies in milk correlated with the total IgA levels $(r=0.39 ; p<0.0001)$. The estimated (geometric mean) value for cow's milk specific IgA on the first day was $14.0 \mathrm{EU} / \mathrm{mL}(95 \% \mathrm{CI}, 7.7-25.3 \mathrm{EU} / \mathrm{mL})$, and the values decayed in 1.5 mo (Fig. 2). Surprisingly, the levels of $\operatorname{IgA}$ antibodies to cow's milk in the milk from the mothers of the infants with CMA and from the healthy controls were comparable.

The levels of total and cow's milk-specific IgA antibodies in mothers' milk were comparable in the mothers of a baby with infantile colic but no CMA (disease control group) and in those with a healthy infant. The contents of total and cow's milkspecific IgA antibodies in human milk did not correlate with maternal atopy.

\section{DISCUSSION}

As demonstrated in the present study, low levels of colostral and human milk IgA may predispose the offspring toward the development of CMA. Further, we found that the low total IgA

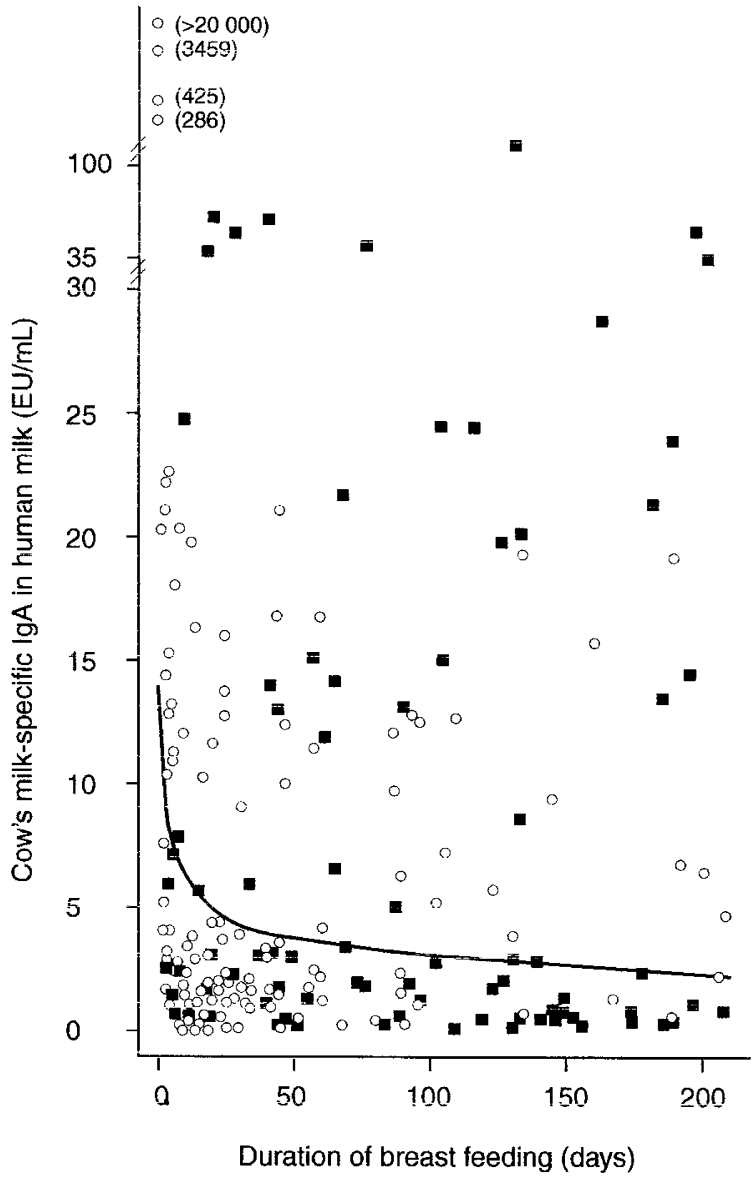

Figure 2. Concentration of $\operatorname{IgA}$ specific to cow's milk in the milk of mothers with a healthy infant (open circles) and in those with a CMA infant (closed squares) as a function of lactational duration. Solid line represents the modeled geometric mean. A common model was used for both groups because the levels of IgA were comparable in the groups.

content of human milk was associated not with maternal atopy but with the allergic status of the infant.

Infants may have allergic reactions even during exclusive breast-feeding (29). The allergic reactions to foods in these infants have been proposed to be caused by passage into human milk of small amounts of proteins ingested by the lactating mother and detectable by immunologic techniques in human milk $(13,27,30)$. In fact, the presence of food proteins in human milk is not an infrequent finding. Beta-lactoglobulin, for example, has been found in milk of $75 \%$ of mothers consuming cow's milk (30). Earlier studies, however, had shown that the quantities of cow's milk proteins or their split products secreted into the milk are not related to the development of CMA (14). This suggested that the cow's milk antigens in human milk were probably not the only factors affecting the induction and maintenance of allergic symptoms of the infant to cow's milk.

Previous studies have established the presence of IgA antibodies to food proteins in human milk (13, 14, 20, 22). Machtinger and Moss (13) detected breast milk IgA to casein or whole milk in $84 \%$ of donors. They found that infants with symptom scores highly suggestive of allergic disease had received human milk with smaller quantities of $\operatorname{IgA}$ antibodies 
to whole cow's milk and casein than infants without clinical manifestations. This was supported by the preliminary finding of Savilahti et al. (14), demonstrating in a small number of infants that, in the mothers whose infants developed CMA manifested by cutaneous symptoms, the milk contained less IgA throughout lactation. This finding was confirmed in our present study with a larger population of CMA infants showing a wide spectrum of symptoms of CMA. Whereas in the study of Savilahti et al. (14), the comparison group consisted of nonatopic mothers with a nonallergic infant, in our study it comprised both atopic and nonatopic mothers with a healthy infant as no association was detected between the maternal atopic history and average total IgA level in the human milk. Savilahti et al. (14) did not find any significant difference in the concentration of cow's milk-specific IgA antibodies between the milk of the mothers whose infants became allergic to cow's milk and that of the comparison group. This finding was supported in the present study.

Several factors, such as SIgA, contribute to the controlled uptake of intestinal antigens from the small intestine. In the newborn period, many of these factors are undeveloped. These sIgA antibodies have been reported to appear in intestinal secretions only after several months of intraluminal antigenic stimulus to local precursors of sIgA-producing plasma cells (31). It is therefore highly likely that in the state of relative sIgA deficiency that occurs during early infancy, foreign proteins may readily gain access to the systemic circulation (24). However, this tendency of the immature intestines of the neonates to allow excessive uptake of foreign antigens across the mucosal barrier is offset by the passive protection of the intestinal surface normally afforded by ingestion of human colostrum (24). Many factors in human milk can facilitate the maturation of the intestinal barrier and at the same time provide secretory antibodies in the gut lumen to provide exclusion of foreign antigens at the intestinal surface (32-34), thus probably lowering the risk of sensitization. A lack of $\operatorname{IgA}$ in milk may lead to an increased exposure of the intestinal mucosa to potential allergens and enhance the risk of food allergies.

In the present study, the total breast milk IgA antibody levels were highest in colostrum, fell rapidly during a 1 - to 2 -wk period, and remained at a stable level of 0.2 to $0.3 \mathrm{~g} / \mathrm{L}$ for several months in both groups of mothers. These values are comparable to the levels found in previous studies $(13,14)$. In the mothers with an infant with CMA, the decline was less pronounced owing to the lower starting values. A higher concentration of SIgA in human milk in the first couple of weeks of lactation may explain why the majority of infants with CMA did not show symptoms suggestive of CMA until an age of 2 to $4 \mathrm{wk}$. In the present study, more milk samples were acquired, as lactation progressed, from those mothers whose infants developed CMA than from those with healthy infants. However, this was taken into account with modeling.

The cause of the observed low IgA content in the milk in the mothers of infants with CMA remains unexplained. According to the present and previous studies, the low IgA content of human milk was not associated with maternal atopy (13). It is well established that human milk sIgA is produced locally in the lactating mammary gland, being elaborated by plasma cells situated proximal to the ductal epithelium. These local plasmacytes are derived from the gut-associated lymphoid tissue, where they have been exposed to specific enteric antigens before being switched to production of IgA isotype and migrating to the breast $(35,36)$. This enteromammary link is directed by lactogenic hormones. There is evidence also that colostral lymphocytes shed into the colostrum are capable of producing $\operatorname{Ig}(36,37)$. In our series, the mothers of infants were verified by a thorough interview not to have been on any prophylactic elimination diets from the beginning of lactation, suggesting that the lower amount of $\operatorname{sgA}$ in these mothers cannot be attributed to less frequent exposure to common sensitizing food antigens in their guts. One might instead suggest that the deficiency of $\operatorname{IgA}$ at one mucosal site, e.g. the mammary gland, probably reflects a more widespread condition in the mother, locally affecting a number of other mucosal sites, such as the gut of the mother.

In conclusion, we present evidence of the protective effect of colostral and human milk IgA antibodies against the development of CMA in the infant. This may be related to providing exclusion of foreign food antigens at the intestinal surface. A low content of $\operatorname{IgA}$ in human milk may predispose the offspring toward the development of food allergies.

Acknowledgments. The authors thank Dr. Kaisu JuntunenBackman for providing the facilities, Dr. Arja Hakulinen for the recruitment of patients and collection of samples and Hanna Oksanen for statistical analysis of the data. We also thank Marjo Leponiemi for her excellent technical assistance.

\section{REFERENCES}

1. Chandra RK, Puri S, Cheema PS 1985 Predictive value of cord blood IgE in the development of atopic disease and role of breast feeding in its prevention. Clin Allergy 15:517-522

2. Businco L, Marchetti F, Pelligrini G, Cantani A, Perlinin R 1983 Prevention of atopy disease in at risk newborns by prolonged breast feeding. Ann Allergy 51:296-299

3. H246šst A, Husby S, Osterballe O 1988 A prospective study of cow's milk allergy in exclusively breast fed infants: incidence, pathogenetic role of early inadvertent exposure to cow's milk formula, and characterization of bovine milk protein in human milk. Acta Paediatr Scand 77:663-670

4. Saarinen UM, Kajosaari M 1995 Breastfeeding as prophylaxis against atopic disease: prospective follow-up study until 17 years old. Lancet 346:1065-1069

5. Lilja G, Dannaeus A, Foucard T, Graff-Lonnevig V, Johansson SGO, Oman H 1989 Effects of maternal diet during late pregnancy and lactation on the development of atopic diseases in infants up to 18 months of age: in vivo results. Clin Exp Allergy 19:473-479

6. Björksten B 1983 Does breast feeding prevent the development of allergy? Immunol Today 4:215-217

7. Atherton DJ 1983 Breast feeding and atopic eczema. BMJ 287:775-776

8. Kovar MG, Serdula MK, Marks JS, Fraser DW 1984 Review of the epidemiologic evidence for an association between infant feeding and infant health. Pediatrics 74(suppl):615-618

9. Cogswell JJ, Alexander J 1982 Breast feeding and eczema/asthma. [letter] Lancet $1: 910-911$

10. Kaplan MS, Solli NJ 1979 Immunoglobulin E to cow's milk protein in breast-fed atopic children. J Allergy Clin Immunol 64:122-126

11. Taylor B, Wadsworth J, Golding J, Butler N 1983 Breast feeding, eczema, asthma, and hay fever. J Epidemiol Commun Health 37:95-99

12. Kramer MS, Moroz B 1981 Do breast feeding and delayed introduction to solid foods protect against subsequent atopic eczema? J Pediatr 98:546-550

13. Machtinger S, Moss R 1986 Cow's milk allergy in breast-fed infants: the role of allergen and maternal secretory IgA antibody. J Allergy Clin Immunol 77:341-347

14. Savilahti E, Tainio VM, Salmenperä L, Arjomaa P, Kallio M, Perheentupa J 1991 Low colostral IgA associated with cow's milk allergy. Acta Paediatr Scand 80:12071213

15. Järvinen K-M, Juntunen-Backman K, Suomalainen H 1999 Relation between weak HLA-DR expression on breast milk macrophages and cow milk allergy in the suckling infant. Pediatr Res 45:76-81

16. Ogra PL, Losonsky GA, Fishaut M 1983 Colostrum-derived immunity and maternalneonatal interaction. Ann N Y Acad Sci 409:82-95 
17. Kenny JF, Bosman MI, Michaels RH 1967 Bacterial and viral coproantibodies in breast-fed infants. Pediatrics 39:202-213

18. Ogra SS, Weintraub D, Ogra PL 1977 Immunologic aspects of human colostrum and milk: III. Fate and absorption of cellular and soluble components in the gastrointestinal tract of the newborn. J Immunol 119:245-248

19. Ammann AJ, Steihm ER 1966 Immune globulin levels in colostrum and breast milk, and serum from formula- and breast-fed newborns. Proc Soc Exp Biol Med 122:1098-1100

20. Hanson LA, Ahlstedt S, Carlsson B, Fällström SP 1977 Secretory IgA antibodies against cow's milk protein in human milk and their possible effect in mixed feeding. Int Arch Allergy Appl Immunol 54:457-462

21. Hanson LA, Ahlstedt S, Andersson B, Carlsson B, Fällström SP, Mellander L, Porras O, Söderström T, Eden CS 1985 Protective factors in milk and the development of the immune system. Pediatrics 75:172-176

22. McClelland DBC, McDonald TT 1976 Antibodies to cow's milk proteins in human colostrum. [letter] Lancet 2:1251-1252

23. Perkkiö M, Savilahti E 1980 Time of appearance of immunoglobulin-containing cells in the mucosa of the neonatal intestine. Pediatr Res 14:953-955

24. Walker WA 1979 Antigen penetration across the immature gut: effect of immunologic and maturational factors in colostrum In: Ogra PL, Dayton D (eds) Immunology of Breast Milk. Raven Press, New York, pp 227-234

25. Walker WA, Wu M, Isselbach KJ, Bloch KJ 1975 Intestinal uptake of macromolecules: III. Studies on the mechanism by which immunization interferes with antigen uptake. J Immunol 115:854-861

26. Isolauri E, Turjanmaa K 1996 Combined skin prick and patch testing enhances identification of food allergy in infants with atopic dermatitis. J Allergy Clin Immunol 97:9-15
27. Järvinen K-M, Mäkinen-Kiljunen S, Suomalainen H 1999 Cow milk challenge via human milk evokes immune responses in infants with cow milk allergy. J Pediatr 135:506-512

28. Knoflach P, Park BH, Cunningham R, Weiser MM, Albini B 1987 Serum antibodies to cow's milk proteins in ulcerative colitis and Crohn's disease. Gastroenterology 92:479-485

29. Isolauri E, Tahvanainen A, Peltola T, Arvola T 1999 Breast-feeding of allergic infants. J Pediatr 134:27-32

30. Sorva S, Mäkinen-Kiljunen S, Juntunen-Backman K 1994 Betalactoglobulin secretion in human milk varies widely after cow's milk ingestion in mothers of infants with cow's milk allergy. J Allergy Clin Immunol 93:787-793

31. Selner JC, Merrill DA, Claman HN 1968 Salivary immunoglobulin and albumin: development during the newborn period. J Pediatr 72:685-689

32. Ammann AJ, Hong R 1971 Selective IgA deficiency: presentation of 30 cases and a review of the literature. Medicine 50:223-236

33. Tomasi TB, Tan EM, Solomon A, Prendergast RA 1965 Characteristics of an immune system common to certain external secretions. J Exp Med 121:101-124

34. Widdowson EM, Colombo VE, Artavantis CA 1976 Changes in the organs of pigs in response to feeding for the first $24 \mathrm{~h}$ after birth. Biol Neonate 28:272-281

35. Hanson LA, Carlsson B, Cruz JR, Garcia B, Holmgren J, Khan SR, Lindblad BS, Svennerholm A-M, Svennerholm B, Urrutia J 1979 Immune response in mammary gland. In: Ogra PL, Dayton D (eds) Immunology of Breast Milk. Raven Press, New York, pp 145-154

36. Slade HB, Schwatz S 1989 Antigen-driven immunoglobulin production by human colostral lymphocytes. Pediatr Res 25:295-299

37. Murillo GJ, Goldman AS 1970 The cells of human colostrum II: synthesis of IgA and beta-1-c. Pediatr Res 4:71-75 